\title{
Transportation and Acceleration of Free Electron by Laser and Economic Monocolor High-Frequency Light Source
}

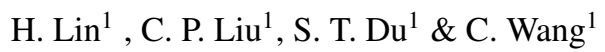 \\ ${ }^{1}$ State Key Laboratory of High Field Laser Physics, Shanghai Institute of Optics and Fine Mechanics, Shanghai \\ Key Laboratory of All Solid-State Laser and Applied Techniques, Shanghai, China \\ Correspondence: H. Lin, State Key Laboratory of High Field Laser Physics, Shanghai Institute of Optics and \\ Fine Mechanics, Shanghai Key Laboratory of All Solid-State Laser and Applied Techniques, P. O. Box 800-211, \\ Shanghai 201800, China. E-mail: linhai@siom.ac.cn
}

\author{
Received: March 8, 2013 Accepted: April 15, 2013 Online Published: May 23, 2013 \\ doi:10.5539/apr.v5n3p123 URL: http://dx.doi.org/10.5539/apr.v5n3p123
}

\begin{abstract}
Strict analysis, based on classic theory, reveals that free electron has an open spiral, instead of familiar closed figure-8, orbit in mono-color electromagnetic wave and does nonlinear oscillation along this open orbit. This implies that the electromagnetic wave can transport a free electron over macroscopic distance and accelerate electron in a limited extent. The transportation and acceleration can lead to an economic setup of monochromatic high-frequency light source.
\end{abstract}

Keywords: monocolor high-frequency, free electron, high-frequency light source

PACS: 41.60.-m 03.50.De 41.20.-q

\section{Introduction}

High-frequency monochromatic light source has attracted broad attention over decades. There are mainly two technical roads to achieve this goal (Winterfeldt, Spielmann, \& Gerber, 2008). One is atomic light source, which is based on laser-driven atomic radiation or so-called high-order harmonics generation (HHG) (Krause, Schafer, \& Kulander, 1992; Corkum, 1993). The other is based on laser-plasma interaction to obtain high-energy electron beams and then obtain their radiations through free-electron-laser (FEL) mechanism (Roberson \& Sprangle, 1991).

The fundamental physics of such a light source is usually interpreted by the behavior of a free electron in a monocolor electromagnetic wave. It is involved in many physical issues, from senior Compton scattering (Sanderson, 1965; Eberly \& Sleeper, 1968; Brown \& Kibble, 1964) to junior atomic high-order harmonics generation (HHG) (Reiss, 2008; Gibbon, 2005). It is also the basis for understanding complicated phenomena taking place in plasmas (Rax, 1992; Gibbon, 1996; Puhkov \& Meyer-ter-Vehn, 1996; Esarey, Ride, \& Sprangle, 1993; Umstadter et al., 1996). A lot of in-depth works have been paid to this topic. By now, it has been acknowledged that the shape of such an orbit is alike to figure-8, (where the long axis of the figure-8 is along the electric field $E$, and the short axis is along the direction of propagation $k$ of the field. The vectors $E, B$, and $k$ are mutually perpendicular) (Eberly $\&$ Sleeper, 1968; Sarachik \& Schappert, 1970; Reiss, 2008). The free electron oscillates along this figure-8 orbit, and the oscillation frequency along the short axis is twice that along the long axis (Reiss, 2008).

This viewpoint is derived from advanced Hamilton-Jacobi equation (Eberly \& Sleeper, 1968; Brown \& Kibble, 1964; Sarachik \& Schappert, 1970). As stated in reference (Eberly \& Sleeper, 1968), the space-dependence of the vector potential is not taken into account. This is the root reason for this viewpoint. It can be verified from easier Newton equation (which is presented in the cylindric frame $(\rho, z, \theta)$ now)

$$
\begin{gathered}
m_{e} d_{t t} \rho=E_{0} \sin \left(-\omega_{0} t\right)-d_{t} z * B_{0} \sin \left(-\omega_{0} t\right) \\
m_{e} d_{t t} z=d_{t} \rho * B_{0} \sin \left(-\omega_{0} t\right) .
\end{gathered}
$$

where $E_{0}$ and $B_{0}$ are two constants. Mathematically, we can verify above-mentioned conclusion from this equation set. Obviously, this equation set does not truly describe the motion of the free electron in electromagnetic fields because the force on the electron is unchanged with respect to its position $(\rho(t), z(t))$. 


\section{Method and Results}

Stricter description on the motion of a free electron should read

$$
\begin{aligned}
m_{e} d_{t t} \rho= & E_{0} \sin \left(k z-\omega_{0} t\right)-d_{t} z * B_{0} \sin \left(k z-\omega_{0} t\right) ; \\
& m_{e} d_{t t} z=d_{t} \rho * B_{0} \sin \left(k z-\omega_{0} t\right)
\end{aligned}
$$

where $k$ meets $k E_{0}=\omega_{0} B_{0}$. By re-writing Equation (2.2) as

$$
d_{t} \rho=\frac{m_{e} d_{t t} z}{B_{0} \sin \left(k z-\omega_{0} t\right)}
$$

and applying $d_{t}$ on it, then, inserting it into Equation (2.1), we can obtain an equation of $\theta=k z-\omega t$ (where $\omega_{B}=\frac{B_{0}}{m_{e}}$ is cyclotron frequency)

$$
d_{t t t} \theta-\operatorname{ctg}(\theta) * d_{t} \theta * d_{t t} \theta+\omega_{B}^{2} \sin ^{2} \theta d_{t} \theta=0,
$$

which means

$$
d_{t t} \theta=\omega_{B}^{2} \cos \theta \sin \theta+C \sin \theta=\frac{1}{2} \omega_{B}^{2} \sin (2 \theta)+C \sin \theta,
$$

or

$$
\frac{1}{2}\left[d_{t} \theta\right]^{2}+\frac{1}{4} \omega_{B}^{2} \cos (2 \theta)+\frac{1}{2} C \cos \theta=D .
$$

Here, constants $D$ and $C$ are determined from following initial conditions:

$$
\begin{gathered}
\theta(t=0)=k z(t=0)=0 ; \\
\left.d_{t t} z\right|_{t=0}=0 ;\left.d_{t t} \theta\right|_{t=0}=0 \\
\left.d_{t} z\right|_{t=0}=0 ;\left.d_{t} \rho\right|_{t=0}=0 \\
\left.d_{t} \theta\right|_{t=0}=-\omega_{0} .
\end{gathered}
$$

From these initial conditions, we have $D=\frac{1}{2} \omega_{0}^{2}+\frac{1}{4} \omega_{B}^{2}+\frac{1}{2} C$ and $C=-\omega_{B}^{2}$ (which arises from $\left.d_{t} \rho\right|_{t=0}=0$ ) hence

$$
d_{t} \theta= \pm \sqrt{\omega_{0}^{2}-\frac{1}{2} \omega_{B}^{2}-\frac{1}{2} \omega_{B}^{2} \cos (2 \theta)+\omega_{B}^{2} \cos \theta}= \pm \sqrt{\omega_{0}^{2}-\omega_{B}^{2} \cos ^{2} \theta+\omega_{B}^{2} \cos \theta}
$$

Some numerical examples are shown in Figures (1-3).

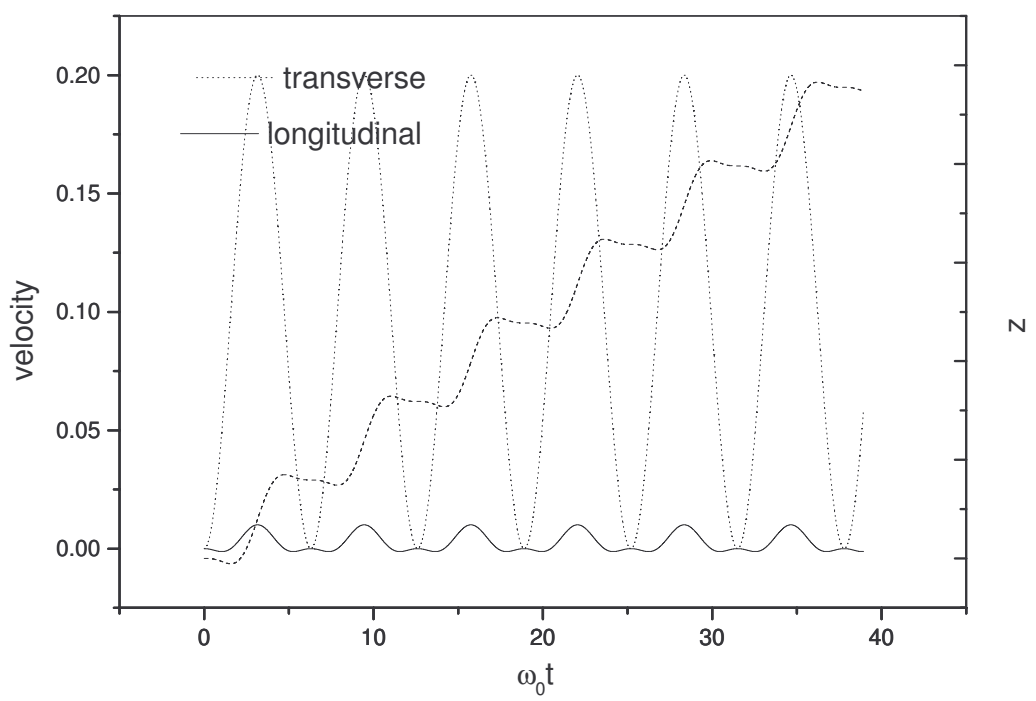

Figure 1. The history of $d_{t} \rho, d_{t} z$, and $z$ 


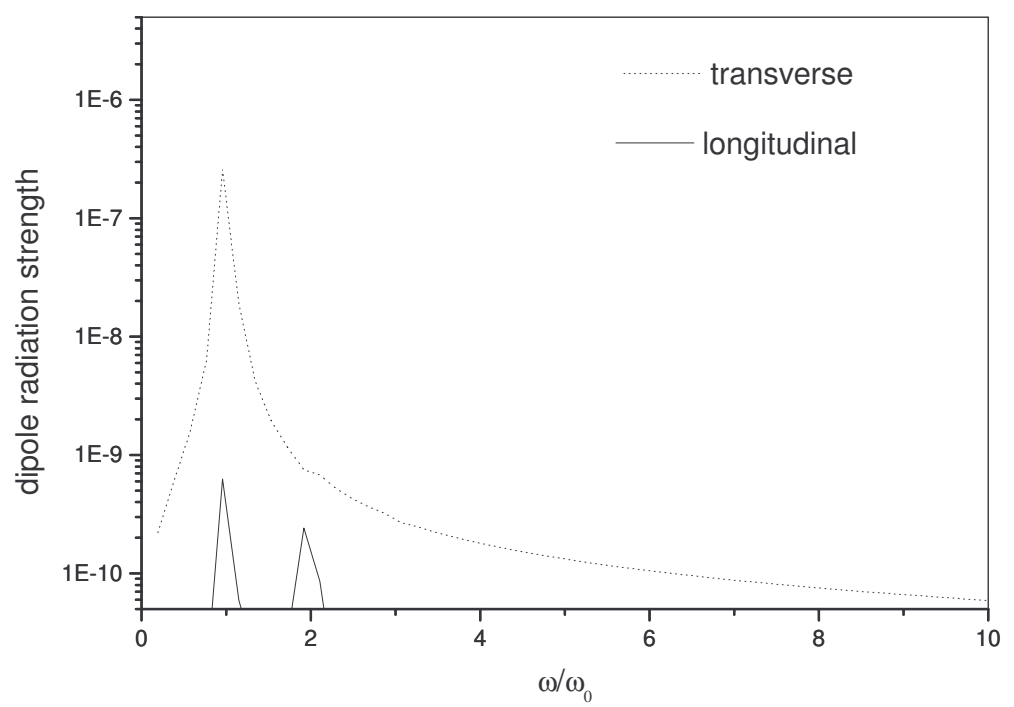

Figure 2. Fourier spectrum of transverse dipole, which is associated with $d_{t t} \rho$, and that of longitudinal one, which is with $d_{t t} z$
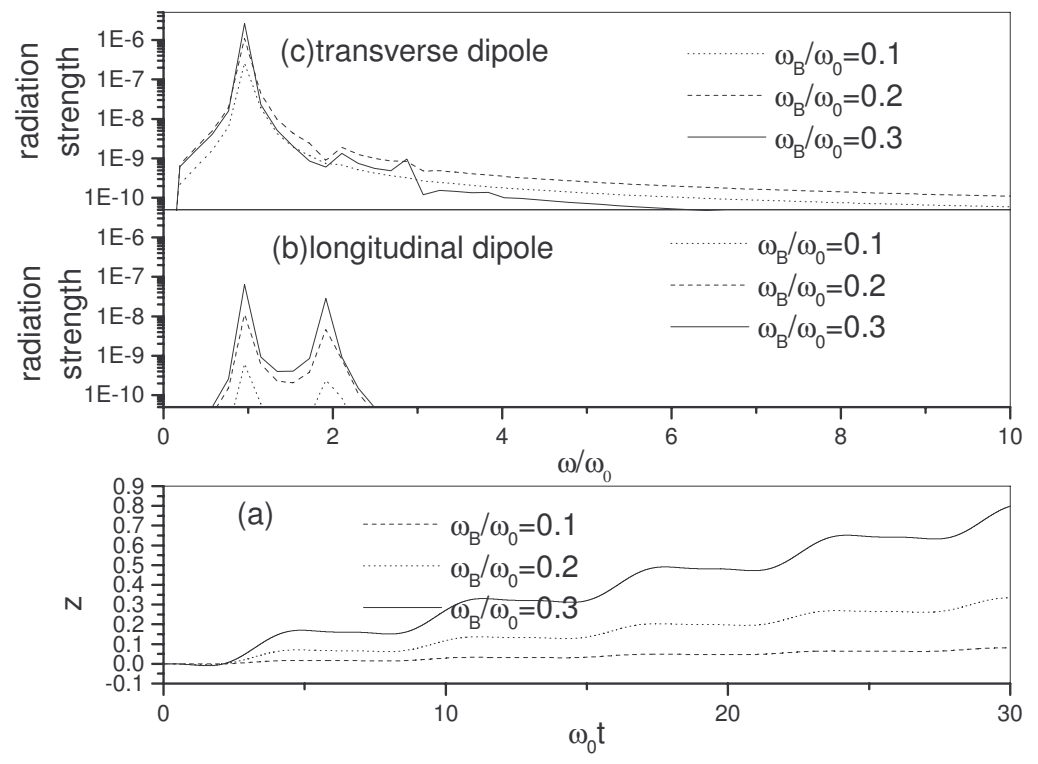

Figure 3. $z(t)$ and Fourier spectrum under different values of $\omega_{B} / \omega_{0}$ 
According to Equation (6) and Figure 1, $\theta(t)$ is a periodic function of $t$ and hence implies that $z(t)$ is not a periodic function of $t$. Likewise, $\rho(t)$ is also not a periodic function of $t$. Therefore, the orbit is not closed and instead open and spiral. On the other hand, both $d_{t} \rho$ and $d_{t} z$ are periodic functions of $t$. Their calculated Fourier spectrum have respective spread. These results indicate that the free electron is pushed forward, along a spiral orbit, by the electromagnetic wave. This alters the conventional viewpoint that the free electron will oscillate along a figure- 8 orbit and its average displacement (relative to its initial position) is 0 .

Equations (1) and Equation (2) are often used to elucidate the underlying physics of atomic light source (Reiss, 2008; Gibbon, 2005). Such a light source is often related with atomic dipole radiation model. The photoelectron is usually viewed as a free electron and the ion is taken as immobile. The calculation on the atomic dipole moment is as same as above described. The dipole moment consists of two perpendicular oscillations. If the oscillating dipole moment have a spectrum spread, photons of higher frequency $>2 \omega_{0}$ will be available from the interaction of a free electron with the electromagnetic wave of a frequency $\omega_{0}$. Figure 2 shows some examples of Fourier spectrum of $d_{t t} \rho$ and $d_{t t} z$. As indicated by these spectrum, the transverse dipole is mainly centered at a $\omega_{0}$-component and the longitudinal one is at $\omega_{0}$-component and $2 \omega_{0}$-component. This differs from the conventional viewpoint that both the transverse oscillation and the longitudinal one are mono-color and their frequencies are $\omega_{0}$ and $2 \omega_{0}$ respectively (Eberly \& Sleeper, 1968; Sarachik \& Schappert, 1970; Reiss, 2008). Moreover, a portion of the dipole radiation energy are at high-frequency $\left(>2 \omega_{0}\right)$ components. These examples imply the capacity of a free electron converting applied electromagnetic energy to higher frequency components. But as shown in Figure 2, the efficiency of such a conversion is too low. In addition, Figure 3 indicates that larger values of the ratio $\omega_{B} / \omega_{0}$ correspond to larger longitudinal displacement of the free electron relative to its initial position. There are little pronounced difference among these spectrum under different values of $\omega_{B} / \omega_{0}$. This implies that it is less effective to enhance the conversion efficiency by increasing $\omega_{B} / \omega_{0}$.

Stricter analysis improves our knowledge on the free electron orbit in a mono-color electromagnetic wave. This is valuable for us to interpret many related phenomena on a sound basis. Otherwise, we might be misguided.

Although such an open orbit is not optimistic to convert radiation to higher-order harmonics components, it enlightens us to pursue more complicated orbit by applying more complicated driving EM pulse. Whether or not more complicated electron orbit can lead to an econormic light source is still an open question. In following paragraph, we continue similar discussions in two typical cases: one includes two color fields and the other is a Gaussian driving pulse.

Likewise, when there are two color fields, the motion reads

$$
\begin{gathered}
m_{e} d_{t t} \rho=E_{1} \sin \left(k_{1} \xi\right)+E_{2} \sin \left(k_{2} \xi\right)-d_{t} z *\left[B_{1} \sin \left(k_{1} \xi\right)+B_{2} \sin \left(k_{2} \xi\right)\right] \\
m_{e} d_{t t} z=d_{t} \rho *\left[B_{1} \sin \left(k_{1} \xi\right)+B_{2} \sin \left(k_{2} \xi\right)\right]
\end{gathered}
$$

where $\xi=z-c t, E_{1,2}$ and $B_{1,2}$ are constants, $k_{1} E_{1}=\omega_{1} B_{1}, k_{2} E_{2}=\omega_{2} B_{2}, \omega_{1,2}=k_{1,2} c$. Following same procedure of deriving Equation (4), we can obtain an equation of $\xi$ (where $\omega_{B 1,2}=\frac{B_{1,2}}{m_{e}}$ are two cyclotron frequencies)

$$
d_{t t t} \xi-\left[\frac{k_{1} \omega_{B 1} \cos \left(k_{1} \xi\right)+k_{2} \omega_{B 2} \cos \left(k_{2} \xi\right)}{\omega_{B 1} \sin \left(k_{1} \xi\right)+\omega_{B 2} \sin \left(k_{2} \xi\right)}\right] * d_{t} \xi * d_{t t} \xi+m_{e}^{2}\left[\omega_{B 1} \sin \left(k_{1} \xi\right)+\omega_{B 2} \sin \left(k_{2} \xi\right)\right]^{2} d_{t} \xi=0
$$

which means

$$
d_{t t} \xi=m_{e}^{2}\left[\omega_{B 1} \sin \left(k_{1} \xi\right)+\omega_{B 2} \sin \left(k_{2} \xi\right)\right]\left[k_{1} \omega_{B 1} \cos \left(k_{1} \xi\right)+k_{2} \omega_{B 2} \cos \left(k_{2} \xi\right)+G\right]
$$

or

$$
\begin{array}{r}
\frac{1}{2}\left[d_{t} \xi\right]^{2}+m_{e}^{2} G\left[\frac{\omega_{B 1}}{k_{1}} \cos \left(k_{1} \xi\right)+\frac{\omega_{B 2}}{k_{2}} \cos \left(k_{2} \xi\right)\right] \\
+\frac{1}{4} m_{e}^{2}\left[\omega_{B 1}^{2} \cos \left(2 k_{1} \xi\right)+\omega_{B 2}^{2} \cos \left(2 k_{2} \xi\right)+2 \omega_{B 1} \omega_{B 2} \cos \left(\left(k_{1}+k_{2}\right) \xi\right)+2 \omega_{B 1} \omega_{B 2} \cos \left(\left(k_{1}-k_{2}\right) \xi\right)\right]=D .
\end{array}
$$

Here, constants $D$ and $G$ are determined from following initial conditions:

$$
\begin{aligned}
& \xi(t=0)=z(t=0)=0 \\
& \left.d_{t t} z\right|_{t=0}=0 ;\left.d_{t t} \xi\right|_{t=0}=0
\end{aligned}
$$




$$
\begin{gathered}
\left.d_{t} z\right|_{t=0}=0 ;\left.d_{t} \rho\right|_{t=0}=0 \\
\left.d_{t} \xi\right|_{t=0}=-c .
\end{gathered}
$$

From these initial conditions, we have $D=\frac{1}{2} c^{2}+\frac{1}{4} m_{e}^{2}\left[\omega_{B 1}^{2}+\omega_{B 2}^{2}+4 \omega_{B 1} \omega_{B 2}\right]+m_{e}^{2} G\left[\frac{\omega_{B 1}}{k_{1}}+\frac{\omega_{B 2}}{k_{2}}\right]$ and $G=-k_{1} \omega_{B 1}-$ $k_{2} \omega_{B 2}$ (which arises from $\left.d_{t} \rho\right|_{t=0}=0$ ) hence

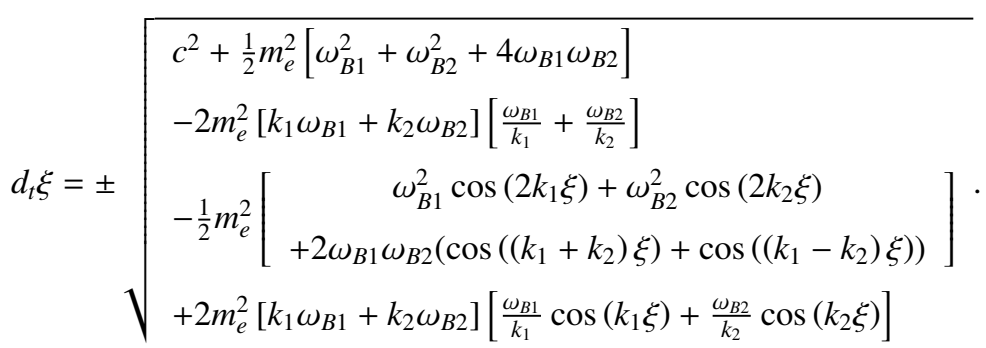

Usually, the driving electromagnetic pulse has a spectrum spread and hence its vector potential a Gaussian longitudinal envelope $A=A_{0} \exp \left(-\frac{\xi^{2}}{W^{2}}\right)$. In this case, we will have

$$
\begin{gathered}
E=E_{0} \xi \exp \left(-\frac{\xi^{2}}{W^{2}}\right)=c B \\
d_{t t t} \xi-\left[\frac{1-2 \xi^{2} / W^{2}}{\xi}\right] * d_{t} \xi * d_{t t} \xi+\omega_{B}^{2} \xi^{2} \exp \left(-\frac{2 \xi^{2}}{W^{2}}\right) d_{t} \xi=0 \\
d_{t t} \xi=-\left[\xi \exp \left(-\frac{\xi^{2}}{W^{2}}\right)\right]\left[\frac{1}{2} W^{2} \omega_{B}^{2} \exp \left(\frac{\xi^{2}}{W^{2}}\right)+G\right] \\
\frac{1}{2}\left[d_{t} \xi\right]^{2}+\frac{1}{4} W^{2} \omega_{B}^{2} \xi^{2}-\frac{1}{2} G W^{2} \exp \left(-\frac{\xi^{2}}{W^{2}}\right)=D
\end{gathered}
$$

where $G=-\frac{1}{2} W^{2} \omega_{B}^{2}$ arises from $\left.d_{t} \rho\right|_{t=0}=0$, and hence $D=\frac{1}{2} c^{2}+\frac{1}{4} W^{4} \omega_{B}^{2}$. The derivations of Equations $(16,17,18)$ are described as above.

The open orbit also means a direct acceleration by the laser. As shown in Equation (8), the electronic longitudinal velocity $d_{t} z$ oscillates between 0 and $v_{\max }=c-\sqrt{c^{2}-\frac{\omega_{B}^{2}}{k^{2}}}$. When an electron leaves the laser of a finite transverse size $\left(E=0\right.$ if $\left.\rho>W_{t}\right)$, its velocity is possible to be a non-zero value $\leqslant v_{\max }$. This revealed the feasiblity of direct acceleration by homogeneous shaped laser even though it is merely a limited acceleration which upgrades $d_{t} z$ from 0 to a non-zero value $\leqslant v_{\max }$. By adjusting laser's transverse width $W_{t}$, we can obtain desirable value of electron velocity when it leaves the laser i.e. $\left.d_{t} \rho\right|_{\rho=W_{t}}$ and $\left.d_{t} z\right|_{\rho=W_{t}}$. This is the preliminary step of setting up a monochromatic light source.

The realistic value of this limited acceleration, or that of open orbit, is that electron can make a long-distance drifting over macroscopic scale. In contrast, the figure- 8 orbit viewpoint means that the electron is bound to its initial position. Whether or not an electron can freely drift is of crucial effect on obtaining high-frequency, mono-color radiation. Many works (Keitel \& Knight, 1995) and above numerical results have suggested that the nonlinear oscillation driven by a laser is of finite efficiency of converting radiation from fundamental frequency (or $\omega_{0}$ ) frequency component to far higher frequency one. Moreover, the radiation associated with this nonlinear oscillation is of broad bandwidth and hence is of poor monochromaticity. Therefore, the so-called re-collision picture, which means an energetic electron is re-captured by an positive ion and hence emits a photon, begins to be attentioned (Corkum, 1993; Lewenstein et al., 1995; Keitel \& Knight, 1995). However, the figure-8 orbit viewpoint bounds people's attention to the ion at the initial position of electron (i.e., so-called parent ion). In this situation, considerable laser energy is spent at ionization of atom. This makes people to believe that strong driving laser seems to be necessary to the up-conversion of radiations to higher frequency components. Namely, the upconversion is less economic. If people are interested in a light source of good monochromaticity, a spending on solid-state optical material which is to filter EM components at undesirable band, is inevitable. 

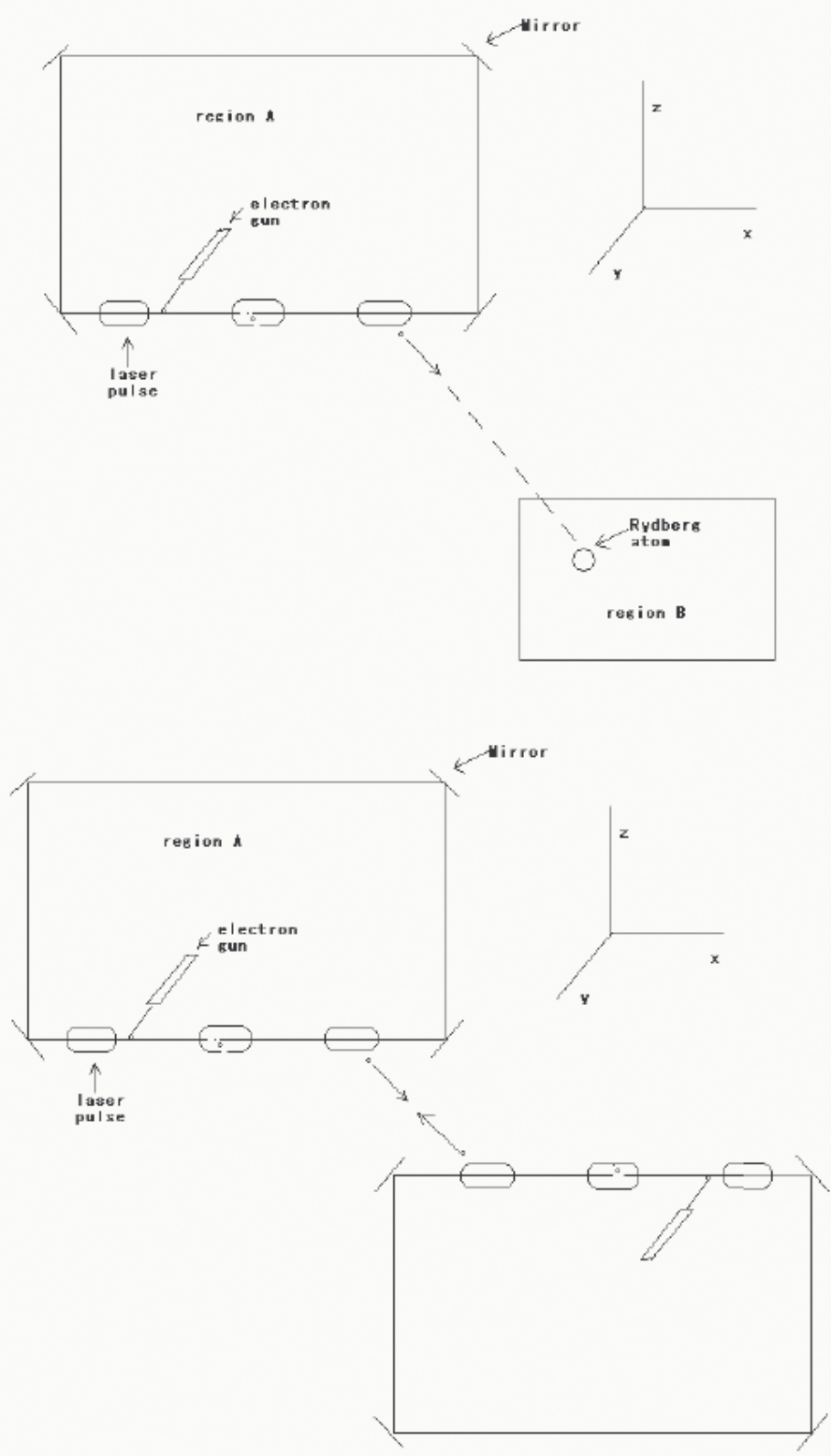

Figure 4. Sketchs of (a)monocolor light source, and (b)electron collider. The small circle represents electron. The light round route is arranged at the $\mathrm{x}-\mathrm{z}$ plane and the electron gun is along the $\mathrm{y}$-axis (or out of the $\mathrm{x}-\mathrm{z}$ plane). Such arrangement is to avoid the cross of the round route and the gun, and hence is favorable to whole device being small-sized

The advantage of the open orbit is to enable an electron to be captured by positive ion and by Rydberg atom at remote position. Thus, good monochromaticity can be achieved even the laser is weak. We can combine a laser device and an low-energy electron gun. For minimizing self-field effect of many electrons, a magnetic device can be used at the exit of the electron gun for picking out fewer electrons interacting with laser. Moreover, the task of the electron gun is merely to supply few electrons into the interaction region and does not demand supplied electrons being of high energies. On the contrary, the supplied electrons are desirous to be of low energies. Low drifting velocity of supplied electron is convenient to adjust the time-of-flight (TOF) of an electron from the exit of the gun to the laser way. Also, it is to cut down unnecessary energy cost and makes the experiments more economic. The direct acceleration can yield an energetic electron leaving the laser. For given laser transverse radius and intensity, the electron's energy when it leaves the laser is determined. A sketch on monocolor light source is illustrated in Figure 4A. The laser acts as a "bus" to transport an electron which is initially at the output of the electron gun to leave its initial position. When the passenger jumps out of the bus, it is along another road 
directed to a positive ion or to a Rydberg atom. The "bus" continues to go forward. Using mirrors, we can set up a round "bus" route and hence repeatedly use the laser. The region A contains the gun and the round route. The output of such a region A are electrons moving in a velocity $\left.d_{t} \rho\right|_{\rho=W_{t}}$ and $\left.d_{t} z\right|_{\rho=W_{t}}$. At the other region (region B), the electron is re-captured and hence emits a photon. Likewise, an electron collider can be set up if we replace the region $B$ with the region A. As shown in Figure 4B, two bus routes and two guns are contained in this electron collider. The electron collider can yield radiations at higher central frequency but of somewhat broad bandwidth.

It is worthy to discuss how to control the electron jump direction when it leaves the laser. Two methods are available. One is to adjust the size of the "bus". As previously discussed, high velocity can be achieved no matter how weak the laser is. Suitable size of laser field can warrant electron's high velocity at the boundary of the laser field. Also, the ratio between transverse velocity and longitudinal velocity at the boundary of the laser field, i.e., the jump direction, is affected by the size of laser field. The transverse size can be controlled by adjusting the width of the fiber transmitting laser. The other method is to adjust the "landing site" of free electrons. Mathematically, the position of the electron when it begins to interact with the laser determines the initial condition in above formulas. For given parameters of the "bus", initial position of the passenger in the "bus" affects his leaving velocity. By adjusting the angle of the electron gun, we can control the distance between the "landing site" and the laser axis, as well as that between the the "landing site" and the laser boundary, and hence following details of the interaction. Comparing two methods, we can find that the latter is simpler and more economic.

\section{Conclusion}

Stricter analysis on the dynamics of free electron in laser field reveals an important property which is covered by approximation theory. According to stricter analysis, the orbit of a free electron in laser field is open rather than our familiar figure- 8 close one. The open orbit enables the free electron to be possible to leave the laser with a high velocity, or to be accelerated by the laser. Because this property does not have special demand on laser strength, it can enlighten us to develop an economic, mono-color light source and economic compact experimental setup for other purposes.

\section{Acknowledgements}

This work is supported by Natural Scientific Fund of China.

\section{References}

Antoine, P., L'Huillier, A., \& Lewenstein, M. (1996). Attosecond Pulse Trains Using High-Order Harmonics. Phys. Rev. Lett., 77, 1234. http://dx.doi.org/10.1103/PhysRevLett.77.1234

Chen, S. Y., Maksimchuk, A., \& Umstadter, D. (1998). Experimental observation of relativistic nonlinear Thomson scattering. Nature, 396(6712), 653. http://dx.doi.org/10.1038/25303

Corkum, P. B. (1993). Plasma perspective on strong field multiphoton ionization. Phys. Rev. Lett., 71, 1994. http://dx.doi.org/10.1103/PhysRevLett.71.1994

Davidson, R. C., \& Uhm, Han S. (1980). Self-consistent Vlasov description of the free electron laser instability. Phys. Fluids, 23, 2076. http://dx.doi.org/10.1063/1.862895

Drescher, M., Hentschel, M., Kienberger, R., Tempea, G., Spielmann, C., Reider, G. A., ... Krausz, F. (2001). X-ray Pulses Approaching the Attosecond Frontier. Science, 291, 1923. http://dx.doi.org/10.1126/science.1058561

Krausz, F. (2001). X-ray Pulses Approaching the Attosecond Frontier. Science, 291, 1923. http://dx.doi.org/10.1126/science.1058561

Eberly, J. H., \& Sleeper, A. (1968). Trajectory and Mass Shift of a Classical Electron in a Radiation Pulse. Phys. Rev., 176, 1570. http://dx.doi.org/10.1103/PhysRev.176.1570

Esarey, E., Ride, S. K., \& Sprangle, P. (1993). Nonlinear Thomson scattering of intense laser pulses from beams and plasmas. Phys. Rev. E, 48, 3003. http://dx.doi.org/10.1103/PhysRevE.48.3003

Gibbon, P. (1996). Harmonic Generation by Femtosecond Laser-Solid Interaction: A Coherent "Water-Window" Light Source? Phys. Rev. Lett., 76, 50. http://dx.doi.org/10.1103/PhysRevLett.76.50

Gibbon, P. (2005). Short Pulse Laser Interactions with Matter-An Introduction. London: Imperial College Press.

Hafizi, B., Sprangle, P., \& Ting, A. (1987). Optical gain, phase shift, and profile in free-electron lasers. Phys. Rev. A, 36, 1739. http://dx.doi.org/10.1103/PhysRevA.36.1739 
Hentschel, M., Kienberger, R., Spielmann, C., Reider, G. A., Milosevic, N., Brabec, T., ... Krausz, F. (2001). Attosecond metrology. Nature, 414, 509. http://dx.doi.org/10.1038/35107000

Jackson, J. D. (1999). Classical Electrodynamics (3rd ed.). New York: Wiley.

Keitel, C. H., \& Knight, P. L. (1995). Monte Carlo classical simulations of ionization and harmonic generation in the relativistic domain. Phys. Rev. A, 51, 1420. http://dx.doi.org/10.1103/PhysRevA.51.1420

Krause, J. L., Schafer, K. J., \& Kulander, K. C. (1992). High-order harmonic generation from atoms and ions in the high intensity regime. Phys.Rev. Lett., 68, 3535. http://dx.doi.org/10.1103/PhysRevLett.68.3535

Kwan, T., Dawson, J. M., \& Lin, A. T. (1977). Free electron laser. Phys. Fluids, $20,581$. http://dx.doi.org/10.1063/1.861916

Landau, L. D., \& Lifshitz, E. M. (1975). Classical Theory of Fields. Oxford, Pergamon.

Lau, Y. Y., He, F., Umstadter, D. P., \& Kowalczyk, R. (2003). Nonlinear Thomson scattering: A tutorial. Physics of Plasmas, 10, 2155. http://dx.doi.org/10.1063/1.1565115

Lewenstein, M., Balcou, Ph., Ivanov, M. Yu. (1994). Phys. Rev. A, 49, 2117. http://dx.doi.org/10.1103/10.1103/PhysRevA.49.2117

L'Huillier, A., \& Balcou, Ph. (1993). High-order harmonic generation in rare gases with a 1-ps 1053-nm laser. Phys. Rev. Lett., 70, 774. http://dx.doi.org/10.1103/PhysRevLett.70.774

Lowell, S. Brown, \& Kibble, T. W. B. (1964). Interaction of Intense Laser Beams with Electrons. Phys. Rev., 133, A705. http://dx.doi.org/10.1103/PhysRev.133.A705

Mairesse, Y., de Bohan, A., Frasinski, L. J., Merdji, H., Dinu, L. C., Monchicourt, P., ... Salires, P. (2003). Attosecond Synchronization of High-Harmonic Soft X-rays. Science, $302,540$. http://dx.doi.org/10.1126/science.1090277

Manheimer, W. M., \& Ott, E. (1974). Theory of microwave generation by an intense relativistic electron beam in a rippled magnetic field. Phys. Fluids B, 17, 463. http://dx.doi.org/10.1063/1.1694738

Paul, P. M., Toma, E. S., Breger, P., Mullot, G., Aug, F., Balcou, Ph., ... Agostini, P. (2001). Observation of a Train of Attosecond Pulses from High Harmonic Generation. Science, 292, 1689. http://dx.doi.org/10.1126/science.1059413

Pfeifer, T., Gallmann, L., Abel, M. J., Neumark, D. M., \& Leone, S. R. (2006). Single attosecond pulse generation in the multicycle-driver regime by adding a weak second-harmonic field. Opt. Lett., $31,975$. http://dx.doi.org/10.1364/OL.31.000975

Pfeifer, T., Kemmer, R., Spitzenpfeil, R., Walter, D., Winterfeldt, C., Gerber, G., \& Spielmann, Ch. (2005). Spatial control of high-harmonic generation in hollow fibers. Opt. http://dx.doi.org/10.1364/OL.30.001497

Pfeifer, T., Walter, D., Winterfeldt, C., Spielmann, Ch., Gerber, G. (2005). Controlling the spectral shape of coherent soft X-rays. Appl. Phys. B, 80, 277. http://dx.doi.org/10.1007/s00340-005-1754-0

Pukhov, A., \& Meyer-ter-Vehn, J. (1996). Relativistic Magnetic Self-Channeling of Light in Near-Critical Plasma: Three-Dimensional Particle-in-Cell Simulation. Phys. Rev. 3975. http://dx.doi.org/10.1103/PhysRevLett.76.3975

Rax, J. M. (1992). Compton harmonic resonances, stochastic instabilities, quasilinear diffusion, and collisionless damping with ultra-high-intensity laser waves. Phys. Fluids B, 4, 3962. http://dx.doi.org/10.1063/1.860299

Reiss, H. R. (2008). Limits on Tunneling Theories of Strong-Field Ionization. Phys. Rev. Lett., 101, 043002. http://dx.doi.org/10.1103/PhysRevLett.101.043002

Roberson, C. W., \& Sprangle, P. (1989). A review of free-electron lasers. Phys. Fluids B, $1,3$. http://dx.doi.org/10.1063/1.859102

Sanderson, J. J. (1965). Corrections to Thompson scattering for intense laser beams. Phys. Letters, $18,114$. http://dx.doi.org/10.1016/0031-9163(65)90674-8

Sarachik, E. S., \& Schappert, G. T. (1970). Classical Theory of the Scattering of Intense Laser Radiation by Free 
Electrons. Phys. Rev. D, 1, 2738. http://dx.doi.org/10.1103/PhysRevD.1.2738

Schafer, K. J., Yang, B., DiMauro, L., \& Kulander, K. C. (1993). Above threshold ionization beyond the high harmonic cutoff. Phys. Rev. Lett., 70, 1599. http://dx.doi.org/10.1103/PhysRevLett.70.1599

Seres, E., Seres, J., Krausz, F., \& Spielmann, Ch. (2004). Generation of Coherent Soft-X-Ray Radiation Extending Far Beyond the Titanium L Edge. Phys Rev Lett., 92, 163002. http://dx.doi.org/10.1103/PhysRevLett.92.163002

Sprangle, P., Tang, C.-M., \& Manheimer, W. M. (1980). Nonlinear theory of free-electron lasers and efficiency enhancement. Phys. Rev. A, 21, 302. http://dx.doi.org/10.1103/PhysRevA.21.302

Umstadter, D., Chen, S. Y., Maksimchuk, A., Mourou, G., \& Wagner, R. (1996). Nonlinear Optics in Relativistic Plasmas and Laser Wake Field Acceleration of Electrons. Science, 273(5274), 472. http://dx.doi.org/10.1126/science.273.5274.472

Winterfeldt, C., Spielmann, C., \& Gerber, G. (2008). Colloquium: Optimal control of high-harmonic generation. Rev. Mod. Phys., 80(1), 117. http://dx.doi.org/10.1103/RevModPhys.80.117

Yamane, K., Zhang, Z. G., Oka, K., Morita, R., Yamashita, M., \& Suguro, A. (2003). Optical pulse compression to $3.4 \mathrm{fs}$ in the monocycle region by feedback phase compensation. Opt. Lett., 28, 2258. http://dx.doi.org/10.1364/OL.28.002258 